\title{
NANOTECNOLOGIA APLICADA AOS COSMÉTICOS
}

\section{NANOTECHNOLOGY APPLIED TO COSMETICS}

\author{
BARIL, M. B'; FRANCO, G. F'; VIANA, R. S'; ${ }^{2} Z A N I N, ~ S . ~ M . ~ W 3$.
}

'Alunas do Programa de Pós-Graduação em Ciências Farmacêuticas da Universidade Federal do Paraná

${ }^{2}$ Farmacêutica. Universidade Federal do Paraná

${ }^{3}$ Professora Associada da Disciplina de Farmacotécnica da Universidade Federal do Paraná

$$
\begin{gathered}
\text { *Autor para correspondência } \\
\text { UFPR - Departamento de Farmácia - Laboratório de Farmacotécnica } \\
\text { Email: sandrazanin@ufpr.br }
\end{gathered}
$$

\section{RESUMO:}

A nanotecnologia está relacionada às estruturas, propriedades e processos envolvendo materiais com dimensões em escala nanométrica. Essas partículas são extensivamente investigadas por promoverem muitas vantagens em relação às formulações tradicionais. A nanotecnologia aplicada à cosmética refere-se à utilização de pequenas partículas contendo princípios ativos que são capazes de penetrar nas camadas mais profundas da pele, potencializando os efeitos do produto. Atualmente existem técnicas distintas para produção e avaliação das nanopartículas, bem como uma grande variedade de polímeros e biopolímeros que são utilizados como matériaprima para o seu desenvolvimento. Embora o mercado seja promissor, ainda é ampla a discussão acerca desta tecnologia uma vez que se encontra em estágio inicial do seu desenvolvimento.

Palavras-chave: cosméticos, pele, nanotecnologia

\section{ABSTRACT:}

Nanotechnology is related to the structures, properties and processes involving materials with nanoscale dimensions. These particles are extensively investigated for promoting many advantages over traditional formulations. Nanotechnology applied to cosmetics refers to the use of small particles containing active ingredients that are able to penetrate into the deeper layers of the skin, increasing the effects of the product. Currently there are different techniques for the production and evaluation of nanoparticles, as well as a wide variety of polymers and biopolymers which are used as raw material for its development. Although the market is promising, is still wide discussion about this technology because it is in the initial stage of its development.

Keywords: cosmetics, skin, nanotechnology

\section{INTRODUÇÃO}

O mercado global vem passando por períodos de grandes competições 
científicas e tecnológicas, principalmente após a nanociência e a nanotecnologia terem sido reconhecidas como uma tendência chave na ciência e tecnologia do século XXI, o que tem ocasionado um aumento nos recursos humanos e financeiros na indústria mundial. A National Science Fundation estima que a nanotecnologia alcançará um impacto na economia global de cerca de 1 trilhão de dólares até 2015, requerendo aproximadamente dois milhões de trabalhadores (RAMOS et al, 2008; NNA, 2005).

O maior nível de desenvolvimento em nanotecnologia é verificado nos Estados Unidos, União Européia e Japão, que investem cerca de um bilhão de dólares ao ano, concentrando juntos cerca da metade dos investimentos no mundo. No entanto, países como a Rússia, China, Índia e Brasil têm realizado investimentos significativos no setor nos últimos anos, sendo que o governo brasileiro já investiu $R$ \$ 140 milhões entre 2001 e 2006 em redes de pesquisa e projetos na área de nanotecnologia (MINISTÉRIO DE CIÊNCIAE TECNOLOGIA, 2006; RAMOS et al, 2008).

A nanotecnologia fundamenta-se na habilidade de caracterizar, manipular e organizar materiais em escala nanométrica. Trata-se de um campo científico multidisciplinar que se aplica a praticamente todos os setores da pesquisa, da engenharia de materiais e processos e de mercado (LEE, 2004; DURÁN et al, 2006). O princípio dessa nova ciência é que os materiais nesta escala nanométrica podem apresentar propriedades químicas, físico-químicas e comportamentais diferentes daquelas apresentadas em escalas maiores (WORLD NANOTECHNOLOGY MARKET, 2005).

A produção de nanocosméticos, especificamente falando, está mundialmente inserida na indústria de cosméticos convencionais, constituindo-se em uma linha de produtos diferenciados de base nanotecnológica, sendo geralmente classificado como um setor específico da indústria química juntamente com os produtos de higiene pessoal e perfumaria (FRONZA et al, 2007).

\section{MATERIAL E MÉTODOS}

A fim de evidenciar o panorama da nanotecnologia no Brasil e no mundo, bem como verificar as suas principais aplicações no setor de cosméticos e as vantagens que é capaz de oferecer aos produtos que utilizam essa tecnologia, realizou-se um levantamento bibliográfico acerca do tema em pesquisa a diversas fontes nacionais e internacionais, tais como teses, dissertações, banco de dados, artigos científicos, livros, periódicos, entre outros.

\section{LEVANTAMENTO BIBLIOGRÁFICO}

Dentre as diversas definições para nanotecnologia possíveis de serem 
encontradas, destaca-se, no âmbito do presente trabalho, aquela utilizada pele Rede de Nanocosméticos, definida pela National Science Foundation como sendo,

(...) o desenvolvimento de pesquisa e tecnologia nos níveis atômico, molecular ou macromolecular na faixa de dimensões entre 1 e 100 nanômetros para fornecer um entendimento fundamental dos fenômenos e materiais na nanoescala e criar e usar estruturas, dispositivos e sistemas que tenham novas propriedades e funções devido ao seu tamanho pequeno ou intermediário. As propriedades e funções novas e diferenciadas são desenvolvidas em uma escala crítica de dimensão da matéria tipicamente abaixo de $100 \mathrm{~nm}(\ldots)$ (FRONZA, 2007).

$\mathrm{Na}$ escala nanométrica, as propriedades dos materiais podem mudar de forma drástica, denominando-se "efeitos quânticos" a essas mudanças. Os átomos passam a revelar características peculiares, podendo apresentar condutividade elétrica, elasticidade, maior reatividade química, maior resistência, cores diferentes, entre outras características, apenas reduzindo o tamanho, sem mudar a substância (GRUPO ETC, 2005).

Tendo como foco o setor cosmético, a empresa pioneira a introduzir um cosmético de base nanotecnológica, no âmbito internacional, foi a Lancôme, divisão de luxo da L’Oréal, em 1995, com o lançamento de um creme para o rosto constituído por nanocápsulas de vitamina $\mathrm{E}$ pura, para combater o envelhecimento da pele. Diversas outras empresas internacionais renomadas também passaram a investir em pesquisa para desenvolver produtos nesta linha (FAPESP, 2008, NEVES, 2008). Empresas como Christian Dior, Anna Pegova, Procter \& Gamble, Revlon, Dermazone Solution, Chanel, Skinceuticals, Estee Lauder, Shiseido, Garnier, Johnsons e Johnsons exemplificam grandes empreendedoras do setor que vieram a lançar produtos baseados em nanotecnologia.

No Brasil, a primeira empresa a desenvolver e colocar no mercado um nanocosmético foi O Boticário, com um creme anti-sinais para a área dos olhos, testa e contorno dos lábios, chamado Nanoserum. A composição nanoestruturada leva ativos como vitamina A, C e K e um produto para clareamento. A tecnologia, desenvolvida em parceria com o laboratório francês Comucel, teve investimentos de R $\$ 14$ milhões e faz parte da linha Active, que começou a ser vendida em 2005. Em 2007, lançou o VitActive Nanopeeling Renovador Microdermoabrasão, cosmético anti-sinais com nanotecnologia aplicada. Outros itens incluem o Liftserum Anti-Sinais e o Sistema Avançado Anti-Sinais 65+. A Natura, por sua vez, lançou em 2007 um produto para hidratação corporal, chamado Brumas de Leite, com partículas da ordem de 150 nanômetros. No mesmo ano também colocou no mercado o Spray Corporal Refrescante para o público masculino (FAPESP, 2008, NEVES, 2008).

A nanotecnologia voltada para a cosmética tem como foco, sobretudo, os produtos destinados à aplicação na pele do rosto e do corpo, com ação 
antienvelhecimento e de fotoproteção, capazes de penetrar nas camadas mais profundas da pele, potencializando os efeitos do produto (NEVES, 2008).

De acordo com Fronza et al (2007) a seguinte definição pode ser aplicada para um nanocosmético: "uma formulação cosmética que veicula ativos ou outros ingredientes nanoestruturados e que apresenta propriedades superiores quanto a sua performance em comparação com produtos convencionais".

No setor cosmético, nanomateriais, como as nanopartículas, estão presentes em xampus, condicionadores, pastas de dentes, cremes anti-rugas, cremes anticelulites, clareador de pele, hidratantes, pós-faciais, loções pós-barba, desodorantes, sabonetes, foto protetores, maquiagens de modo geral, perfumes e esmaltes (FRONZA et al, 2007). As nanoemulsões, por sua vez, constituem uma classe de emulsões com gotículas uniformes e de dimensões muito diminutas, na faixa entre 20 e 500 nm, que estão se tornando cada vez mais populares como veículos para a liberação controlada e dispersão otimizada de ingredientes ativos (CAPEK, 2004).

Em se tratando de produtos cosméticos, entretanto, é necessária uma escolha cuidadosa do tipo de carreador a ser utilizado para uma determinada substância ativa, tendo em vista o objetivo a que seu uso se propõe. Produtos que se destinam a permanecer na pele, sem que ocorra sua absorção, como é o caso dos filtros solares, por exemplo, devem ser formulados para atenderem a esse fim. Dessa forma, a melhor forma de aumentar o desempenho de um ativo em uma formulação cosmética é o desenvolvimento de sistemas de liberação apropriados (MORGANTI et al, 2001).

Alvarez-Román e colaboradores (2004) investigaram a penetração cutânea passiva e a permeação de produtos altamente lipofílicos, como o protetor solar octilmetoxicinamato (OMC) e o corante fluorescente Nile red (NR), encapsulados em nanopartículas biodegradáveis de poli(£)caprolactona, comparativamente aos mesmos produtos não encapsulados. Os autores observaram que o encapsulamento nanoparticulado produziu um aumento de 3,4 vezes no nível de OMC dentro do estrato córneo, embora o uso de nanopartículas não tenha propiciado aumento na permeação do mesmo na pele, mas sim um aumento na sua disponibilidade no estrato córneo. Em nanopartículas contendo NR, a fluorescência foi perceptível a profundidades maiores (acima de $60 \mu \mathrm{m}$ ) dentro da pele, comparativamente quando dissolvido em propilenoglicol, e que a alteração de distribuição foi devida, ao menos em parte, a atividade termodinâmica alterada que resultou em um aumento de seu coeficiente de partição no estrato córneo.

Na figura 1 estão evidenciadas as camadas da pele com suas respectivas dimensões, com base em trabalho de Chien (1992), permitindo a visualização dos diferentes graus de penetração de produtos nanoparticulados na pele. 

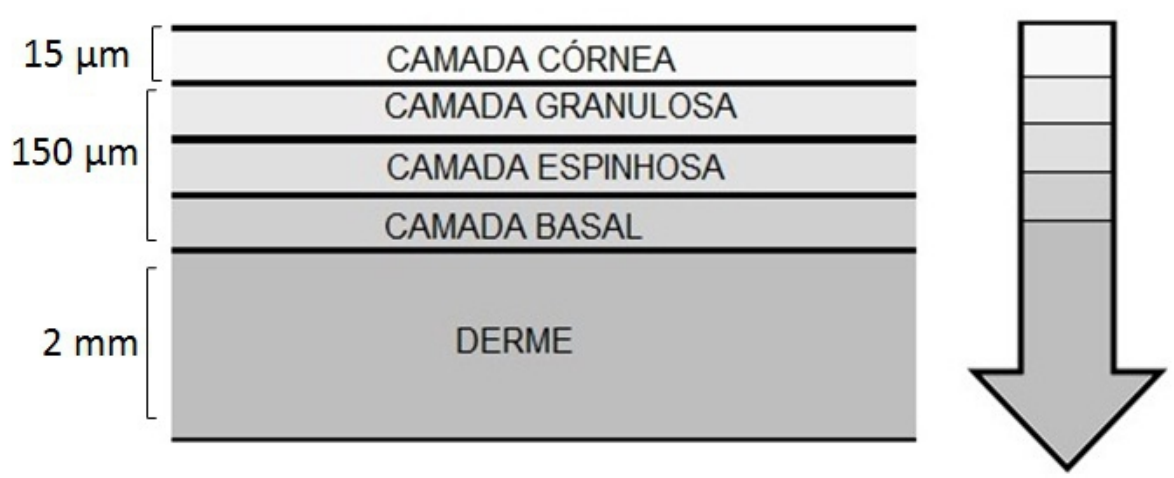

EPIDERME

Figura 1 - Camadas da Epiderme FONTE: Autores (2011)

Por outro lado, quando se pretende um maior grau de penetração podem ser utilizados certos ativos para atender a esta finalidade; é neste âmbito que também se aplicam as nanopartículas. Os primeiros produtos que prometiam combater as rugas, tomando-os como exemplo, eram limitados a esfoliar a área mais superficial da epiderme, a camada córnea. Na década de 70, surgiram cremes cujas formulações continham substâncias que conseguiam penetrar na pele, porém apenas na camada córnea. Já nos anos 80 , surgiram os alfa-hidroxiácidos, com capacidade de penetração um pouco maior. Em 1990, surgiram os lipossomas, minúsculas partículas compostas de gordura e água, que chegavam ainda mais fundo na pele, mas não na camada basal (NEVES, 2008).

Pesquisas realizadas pela Faculdade de Engenharia Química da Unicamp demonstram que atualmente vêm sendo dada uma maior ênfase a dermocosméticos com ação diferenciada, como é o caso dos nanocosméticos, em que se espera, por exemplo, uma ação mais eficaz em rugas e preenchimentos, pela penetração mais profunda das partículas na pele, sem o risco de alcançar a corrente sanguínea. Isto porque, quando as moléculas dos princípios ativos dos cremes possuem tamanhos maiores elas ficam apenas na superfície da pele, protegendo-a da perda de água, tendo efeito puramente cosmético (FAPESP, 2008).

Alvarez-Román e colaboradores (2004), em outro trabalho, utilizando nanopartículas de poliestireno não biodegradáveis e fluorescentes, com diâmetros de 20 e $200 \mathrm{~nm}$, observaram que as nanopartículas se acumularam preferencialmente nas aberturas foliculares e que a sua distribuição aumentou de maneira tempodependente. Verificou-se também que a localização folicular foi favorecida pelas partículas de tamanho menor e que, independentemente desta localização, na região interfolicular também apareciam nanopartículas em imagens de superfície. Contudo, em imagens de secção transversal, observou-se que a estrutura da pele entre os folículos não oferece uma via de penetração alternativa para os vetores poliméricos, 
cujo transporte foi claramente impedido pelo estrato córneo.

As vantagens do uso da nanobiotecnologia na produção de nanocosméticos e formulações dermatológicas advêm da proteção dos ingredientes quanto à degradação química ou enzimática, do controle de sua liberação, principalmente no caso de irritantes em altas doses, e do prolongamento do tempo de residência dos ativos cosméticos ou fármacos na camada córnea (FRONZA et al, 2007).

Segundo Sonia Tuccori, doutoura em Química que trabalha com a área de nanotecnologia na empresa Natura, "Os produtos cosméticos nano têm três apelos irresistíveis: melhor absorção, ação prolongada e um toque mais leve" (RANGEL, 2008). Além disso, novas nuances são fortes tendências, como explica a pesquisadora Silvia Guterres, da Rede de Nanocosméticos, que acredita que "serão obtidas tonalidades de cores nunca vistas antes, com muito mais nuances" (FAPESP, 2008).

Além destas características, Wissing e Müller (2003) também destacam que as nanopartículas são vantajosas para aplicações cosméticas por atuarem como agentes oclusivos e também pelo potencial bloqueador das radiações ultra-violeta, atuando como filtros físicos, podendo estar combinados a filtros químicos com o propósito de melhorar a fotoproteção.

A principal aplicação de nanopartículas poliméricas está justamente focada no desenvolvimento de novas formulações contendo filtro solares, uma vez que estas nanopartículas são capazes de carrear substâncias altamente lipofílicas e por sua capacidade em alterar e/ou mascarar as propriedades físico-químicas de fármacos ou ativos cosméticos encapsulados (OLVERA-MATÍNEZ et al, 2005; GUTERRES et al, 2007).

Nos protetores solares, as gotículas são pigmentos de um branco brilhante que refletem luz de todos os comprimentos de onda. Porém, nanopartículas de $\mathrm{TiO}_{2}$ não refletem a luz visível por serem transparentes, porém ainda bloqueiem a luz UV. Assim, partículas de $\mathrm{TiO}_{2}$ em nanoescala proporcionam excelente proteção UV nas aplicações de filtro solar. Portanto, na forma de nanopartículas, além da maior eficiência, não há o aspecto esbranquiçado típico provocado pela luz espalhada após a aplicação do protetor (NEVES, 2008; TOMA, 2004).

Nas nanoemulsões, por sua vez, as minúsculas dimensões das gotículas reduzem muito a força da gravidade, evitando que haja a criação de sedimentos durante o armazenamento do produto. O pequeno tamanho das gotículas também evita a floculação. Evitando a floculação, o sistema mantém-se disperso, sem separação. As gotículas também evitam a coalescência por não serem deformáveis e não apresentarem alterações da superfície (NEVES, 2008). Outras vantagens das nanoemulsões podem ser observadas no Quadro 1. 
QUADRO 1 - Vantagem das nanoemulsões frente às emulsões tradicionais

\begin{tabular}{|c|l|}
\hline Penetração na pele & $\begin{array}{l}\text { Devido a suas dimensões reduzidas, as nanoemulsões podem } \\
\text { penetrar na superfície da pele, melhorando a penetração de } \\
\text { ingredientes ativos. }\end{array}$ \\
\hline Estética & $\begin{array}{l}\text { A característica transparência do sistema, sua fluidez (em } \\
\text { concentrações razoáveis de óleo), bem como a ausência de } \\
\text { espessante conferem às nanoemulsões ótimo aspecto estético } \\
\text { e agradável sensorial à pele. }\end{array}$ \\
\hline $\begin{array}{c}\text { Uso de menos } \\
\text { tensoativo }\end{array}$ & $\begin{array}{l}\text { Ao contrário das microemulsões, que exigem alta concentração } \\
\text { de tensoativos, normalmente na faixa de 20\% ou mais, as } \\
\text { nanoemulsões podem ser preparadas usando concentração } \\
\text { mais baixa. Para uma nanoemulsão óleo-em-água a 20\%, pode } \\
\text { ser suficiente a concentração de tensoativo da ordem de 5 a }\end{array}$ \\
\hline $\begin{array}{c}\text { Liberação de fragrância } \\
\text { inconanoemulsões podem ser usadas para liberar fragrâncias } \\
\text { incorporadas aos produtos cosméticos. Além disso, matérias- } \\
\text { primas de fragrâncias, como ésteres, aldeídos e cetonas, que } \\
\text { não contêm álcool, podem ser usadas nas formulações de } \\
\text { nanoemulsões. }\end{array}$ \\
\hline $\begin{array}{c}\text { Substituição de } \\
\text { lipossomas }\end{array}$ & $\begin{array}{l}\text { As nanoemulsões podem ser aplicadas como substitutas de } \\
\text { lipossomas e vesículas, sendo possível, em certos casos, } \\
\text { formar fases cristalinas lamelares de líquido ao redor das } \\
\text { gotículas da nanoemulsão. }\end{array}$ \\
\hline
\end{tabular}

FONTE: Adaptado de NEVES (2008).

Em matéria publicada na revista Cosmetics \& Toiletries (2007), Valcinir Bedin (médico dermatologista) afirmou que trabalhos realizados com alguns centros de pesquisa e desenvolvimento na área capilar revelaram que produtos usados para a coloração e para a modificação química da forma dos cabelos (alisamento ou permanentes) poderiam contar com a nanotecnologia. Na coloração dos cabelos há necessidade de abrir demasiadamente as cutículas dos fios a fim de promover a oxidação da melanina. Com a aplicação posterior do novo pigmento ocorre um dano ainda maior, deixando os fios mais ressecados devido à perda de água. Portanto, se a cutícula não for fechada adequadamente pode ocorrer quebra dos fios devido à fragilização da haste. Como a nanotecnologia se utiliza de moléculas muito pequenas, estes danos podem ser evitados, pois é capaz de promover a permeação da tintura sem abrir as cutículas.

Em estudo realizado pela Universidade Estadual de Campinas e a Chemyunion, foram empregadas nanopartículas de sericina no tratamento cosmético de cabelos, culminando com o lançamento do produto Seriseal. A sericina é uma das proteínas constituintes do bicho-da-seda, e possui a capacidade de ligar-se à queratina da pele e cabelos, formando um filme resistente, hidratante e protetor, proporcionando a selagem das cutículas dos fios danificadas (MARCELINO et al, 2008; FAPESP, 2008). 


\section{CONCLUSÃO}

A nanotecnologia é um fenômeno recente e vêm sendo mais extensivamente estudada e regulamentada principalmente nas duas últimas décadas. Esta tecnologia está promovendo uma revolução científica e tecnológica de proporções ainda não totalmente conhecidas.

O setor cosmético vem fazendo uso desta tecnologia devido às diversas vantagens da sua aplicação, principalmente no que concerne a uma maior capacidade de penetração dos ativos nas camadas da pele. Porém, apenas em um futuro próximo, com um maior e mais efetivo desenvolvimento desta tecnologia, é que se poderá ver com mais clareza seus reais benefícios e a segurança dos produtos oferecidos com este apelo. Os possíveis riscos na aplicação de nanopartículas incluem uma possível toxicidade e uma possível ausência de biocompatibilidade dos materiais utilizados. Não menos importante é considerar os impactos ambientais que também pode vir a ocasionar, caso este aspecto não seja alvo de estudos.

\section{REFERÊNCIAS}

ALVAREZ-ROMÁN, R.; NAIK, A.; KAILA, Y. N.; GUY, R. H.; FESSI, H. Enhancement of Topical Delivery from Biodegradable Nanoparticles. Pharmaceutical Research, v. 21, n. 10, p. 1818-1825, 2004.

ALVAREZ-ROMÁN, R.; NAIK, A.; KAILA, Y. N.; GUY, R. H.; FESSI, H. Skin penetration and distribution of polymeric nanoparticles. Journal of Controlled Release, v. 99, p. 53-62, 2004.

BEDIN, V. Nanotecnologia e cabelos: até onde vamos? Cosmetics and Toiletries, v. 19, n. 2, mar-abr, p. 44, 2007.

CAPEK, I. Degradation of Kinetically-stable o/w emulsions. Advances in Colloid Interfacial Science, Amsterdam, v. 107, p. 125-55, 2004.

CHIEN, Y. W. Novel Drug Delivery Systems. New York: Marcel Dekker Inc, p. 310, 1992.

DURÁN, N.; DE AZEVEDO, M. M. M. O que é nanobiotecnologia? Atualidades e perspectivas. Instituto de Química, Laboratório de Química Biológica, Unicamp, Campinas, 2004. 
FRONZA, T.; GUTERRES, S.; POHLMANN, A.; TEIXEIRA, H. Nanocosméticos: Em Direção ao Estabelecimento de Marcos Regulatórios. Porto Alegre: UFRGS, 2007.

GRUPO ETC - Grupo de Ação Sobre Erosão, Tecnologia e Informação (Org). Nanotecnologia: os riscos da tecnologia do futuro: saiba sobre produtos invisíveis que já estão no nosso dia-dia e o seu impacto na alimentação e na agricultura. Porto Alegre: L\&pm, 2005. 197 p.

GUterRes, S.; AlVES, M. P.; POHLMANN, A. R. Polymeric Nanoparticles, Nanosphere and Nanocapsules, for Cutaneous Applications. Drug Target Insights, v. 2, p. 1-11, 2007.

LEE, V. H. L. Nanotechnology: challenging the limit of creativity in targeted drug delivery. Advanced Drug Delivery Reviews, v. 56, p.1527-1528, 2004.

MARCELINO, A.G.; ROSSAN, M. R.; SANTANA, M. H. A.; SILVEIRA, J. E. S.; PEREDA, M. D. V.; NOGUEIRA, C. Nanopartículas de Sericina no Tratamento Cosmético de Cabelos. Cosmetics \& Toiletries, vol. 20, nov-dez, p. 60, 2008.

MINISTÉRIO DE CIÊNCIA E TECNOLOGIA, 2006. Relatório Nanotecnologia: Investimentos, Resultados e Demandas. Disponível em: <http://www.mct.gov.br/upd_blob/0008/8075.pdf>Acesso em: maio de 2010.

MORGANTI, P; RUOCCO, E; WOLF, R; RUOCCO, V. Percutaneous absorption and delivery systems. Clinics in Dermatology, v. 19, p. 489-501, 2001.

NEVES, K. Nanotecnologia em cosméticos. Cosmetics \& Toiletries, v. 20, jan-fev, p. $22,2008$.

NNA, 2005. Nanotechnology news archive. Disponível em: <http://www.azonano.com/news_archived.asp>Acesso em agosto de 2010.

OLVERA-MATÍNEZ, B.; CÁZARES-DELGADILLO, J.; CALDERILLA-FAJARDO, S.; VILLALOBO-GARCÍA, R.; GANEM-QUINTANAR, A.; QUINTANAR-GUERRERO, D. Prepartion af polymeric nanocapsules containing octyl methoxycinnamate by the emulsification-diffusion technique: penetration across the stratum corneum. Journal of Pharmaceutics Sciences, v. 94, n. 7, p. 1552-1559, 2005.

RAMOS, B. G. Z; PASA, T. B. C. O desenvolvimento da nanotecnologia: cenário 
mundial e nacional de investimentos. Rev. Bras. Farm., v. 89 (2): p. 95-101, 2008.

RANGEL, R. O mundo maravilhoso (e promissor) das partículas anãs. Revista Inovação em Pauta, n4, 2008.

TOMA, H.E. O mundo nanométrico: a dimensão do novo século. São Paulo: Oficina de Textos, 2004. 102 p.

WISSING, S. A.; MÜLLER, R. H. Cosmetic applications for solid lipid nanoparticles (SLN). International Journal of Pharmaceutics, v. 254, p. 65-68, 2003.

WORLD NANOTECHNOLOGY MARKET. An industry update. RNCOS. p.1-60, 2005. 